\title{
La retraite des salariés du secteur privé en France
}

Conjuguer redistribution, complexité et incertitude temporelle

The French Public Pensions System. Combining Redistribution, Complexity and Temporal Uncertainty

\section{Karine Briard}

\section{(2) OpenEdition Journals}

Édition électronique

URL : http://journals.openedition.org/travailemploi/7372

DOI : 10.4000/travailemploi.7372

ISSN : 1775-416X

Éditeur

DARES - Ministère du Travail

Édition imprimée

Date de publication : 1 janvier 2017

Pagination : 17-41

ISSN : 0224-4365

Référence électronique

Karine Briard, «La retraite des salariés du secteur privé en France», Travail et Emploi [En ligne],

149 | janvier-mars 2017, mis en ligne le 11 juillet 2019, consulté le 06 septembre 2019. URL : http:// journals.openedition.org/travailemploi/7372 ; DOI : 10.4000/travailemploi.7372 


\title{
La retraite des salariés du secteur privé en France Conjuguer redistribution, complexité et incertitude temporelle"
}

\author{
Karine Briard ${ }^{* *}$
}

\begin{abstract}
Au cours de ses soixante-dix années d'existence, le système de retraite français a affirmé sa mission redistributive et couvre aujourd'hui une large variété de situations en marge de l'emploi. Cette évolution amorcée dans les années 1970 s'est réalisée par à-coups, parfois à contretemps des évolutions socioéconomiques. Si l'architecture et les grands objectifs du système ont persisté, les logiques ont changé : à compter des années 2000, le cadre normatif a fait place à des règles de détermination des droits à retraite plus flexibles, prenant mieux en compte la diversité des carrières et les choix individuels. Le reparamétrage des règles existantes et la superposition de mesures entamés en 1982 ont cependant engendré un système obscur à l'efficacité discutable. L'objectif de justice sociale s'est ainsi parfois heurté à la complexité d'un système aux résultats ambigus et incertains. Cette difficulté à apprécier dans quelle mesure le système assure ses diverses missions peut expliquer la trajectoire de réforme qui a été suivie.
\end{abstract}

L e système de retraite obligatoire des salariés du secteur privé s'est construit dans un contexte de profondes évolutions socio-économiques et démographiques. Ses soixante-dix années d'existence ont été marquées par plusieurs réformes et des révisions plus ponctuelles. Si les grands principes de détermination des droits à retraite n’ont pas été modifiés, les conditions d'acquisition et de valorisation de ces droits ont en revanche notablement évolué. Depuis le début des années 1970, la retraite des salariés du secteur privé comprend au moins deux étages : une pension de base versée par le régime général de la Sécurité sociale, qui est calculée à partir du nombre d'annuités d'assurance et des

\footnotetext{
* L'auteure remercie pour leurs nombreuses remarques Thomas Amossé, Mathieu Grégoire, Jean-Michel Hourriez, Véronique Rémy ainsi que les trois évaluateurs anonymes de l'article.

** Direction de l'animation de la recherche, des études et des statistiques (Dares), Mission animation de la recherche (MAR) ; karine.briard@travail.gouv.fr
} 
salaires de l'assuré, et une retraite complémentaire, qui est calculée en points, en fonction des cotisations acquittées durant sa carrière. Ces principes sont restés inchangés jusqu'à aujourd'hui, mais la sobriété des règles d'origine, qui s'articulaient autour d'un standard de carrière continue, a laissé place à des règles complexes visant à limiter les pertes de droits à retraite pour une grande variété de situations en marge de cette norme. Au cours de la décennie 1970, plusieurs mesures ont été prises pour accorder des droits durant les périodes d'interruption involontaire d'emploi (chômage indemnisé et maladie, en particulier) ainsi qu'aux mères. Bien que les dispositifs mis en place aient pour la plupart été élargis depuis, leur forme est restée inchangée. Les périodes de chômage, par exemple, continuent de donner lieu à la validation de trimestres d'assurance. Indépendamment de ces dispositifs explicites de solidarité, les règles d'attribution et de calcul de la pension portent en elles-mêmes des mécanismes modérant les effets d'aléas de carrière et/ou de faibles rémunérations. Mais leurs effets sont complexes à appréhender et les modifications de paramètres tels que les bornes d'âge, le nombre d'annuités requises pour une pension à taux plein et le nombre de salaires retenus ont accentué cette difficulté. De l'avis des experts publics en charge de son évaluation, le système actuel remplit globalement les missions qui lui ont été assignées (CoR, 2013), dont celle d'offrir aux retraités un niveau de vie proche en moyenne de celui des actifs et d'assurer une solidarité entre ses assurés. Cependant, il ressort aussi que la combinaison de certaines règles de calcul produit des effets inverses à ceux souhaités, qui sont à la défaveur des personnes ayant eu des carrières discontinues et/ou à bas salaires. L'inefficacité du système à traiter de façon équitable les assurés selon leur parcours professionnel interroge ainsi sur la raison d'être de ces règles et sur la possibilité de corriger leurs effets négatifs. Elle invite notamment à examiner comment le système s'est ajusté face à la déstandardisation et à la diversification des parcours professionnels au cours des dernières décennies.

Les analyses récentes de l'évolution des systèmes de protection sociale en Europe continentale mettent en avant le caractère séquentiel et incrémental des changements (STREECK, THELEN, 2005) : les évolutions socio-économiques conduisent à une dérive des systèmes au regard de leurs missions puis, par sédimentation, les modifications successives des règles entraînent une transformation des systèmes eux-mêmes. L'une des caractéristiques des systèmes de retraite par rapport aux autres institutions de protection sociale est le cadre de long terme dans lequel ils s'inscrivent : les droits à retraite sont valorisés plusieurs décennies après avoir été accordés et une modification des règles peut affecter différemment les assurés selon le moment où ils sont concernés. La complexité des liens entre les règles de calcul et leurs effets, à laquelle s'ajoute la multiplicité des objectifs poursuivis par les systèmes publics, rend ainsi la politique

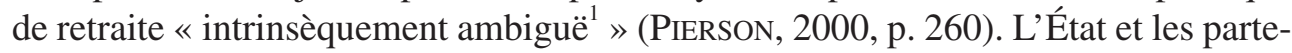
naires sociaux ne disposent souvent que d'une information limitée pour décider des orientations à suivre et prendre les dispositions adéquates pour corriger des défaillances qu'ils ont identifiées ${ }^{2}$. Or, si les problématiques de retraite sont considérées par les

1. Notre traduction, "inherently ambiguous" dans le texte.

2. Sur l'information limitée en matière de politique publique, voir par exemple WITT (2003) et MuLLER (2000). 
décideurs publics comme lointaines et difficiles à circonscrire, leur mise à l'agenda politique est ajournée, ce qui a pour conséquence de laisser persister, voire d'accentuer, les discordances entre le système et les situations qu'il est censé prendre en charge. Les régimes de retraite en annuités, dans lesquels la valeur des droits accordés n'est déterminée qu' au terme de la carrière, tendent à renforcer ces effets de long terme et l'incertitude temporelle qui leur est associée. Leurs capacités d'ajustement limitées ont fait naître de nombreuses propositions de réformes, s'accordant pour la plupart sur des règles plus flexibles renforçant le lien entre activité professionnelle et retraite (FELDSTEIN, SIEBERT, 2002). Au-delà du principe, la variété des solutions effectivement adoptées par les pays, allant d'une modification des paramètres d'ouverture des droits (relèvement des âges légaux de départ, augmentation de la durée de cotisation) comme en Espagne ou en Belgique, à des changements plus structurels conduisant à l'abandon des annuités comme en Italie ou en Suède, traduit cependant la singularité de l'évolution de chaque système (PIERSON, 2000). En France, les réformes qui se sont succédé, toutes paramétriques, ont maintenu la complémentarité entre les fonctionnements en annuités et en points. Cette stabilité des grands principes de détermination des droits dans un contexte de profondes transformations socio-économiques interroge sur le degré d'ajustement du système de retraite. L'attachement aux annuités et le choix de ne réformer que les paramètres n'ont-ils pas conduit à un déphasage du système avec les situations individuelles ? Les réformes du système ont-elles réussi à contrer sa dérive pour préserver ses missions ? Les valeurs et les normes qu'il porte ont-elles été modifiées ? Les défaillances actuelles du système par rapport à ses objectifs questionnent en outre sur le processus de réforme. Résultent-elles d'un biais de perception, d'erreurs de jugement, de retards dans la définition de la politique de retraite ? Si le rythme et la nature des ajustements ont été guidés par un apprentissage du fonctionnement du système et de ses interactions avec le contexte économique, n'est-ce pas le signe que les instruments d'ajustement sont insuffisants et restent à parachever? Si les règles mêmes sont la cause de certains dysfonctionnements, faut-il alors les repenser ?

Pour éclairer l'ensemble de ces questions, cet article cherche à apprécier dans quelle mesure les évolutions du système de retraite des salariés du secteur privé ont visé et respecté un traitement équitable des assurés s'éloignant de la norme de carrière continue. En considérant les décisions techniques prises successivement, il s'interroge en particulier sur la faculté des acteurs institutionnels à pouvoir faire des choix éclairés pour ajuster le système à cet objectif, à disposer d'une information suffisante sur la situation du système et de la population assurée, et à l'utiliser ${ }^{3}$. L'article s'appuie sur l'évolution de la législation vieillesse ${ }^{4}$, des travaux d'évaluation et notre propre expérience, de 2001 à 2014, en tant qu'économiste à la Caisse nationale d'assurance vieillesse (Cnav ; gestionnaire de la retraite de base des salariés du privé), puis chargée

3. Nous n'abordons que les difficultés d'accès à l'information factuelle, sans aborder celles portant sur les stratégies des acteurs ou la demande sociale, comme ce peut être le cas en analyse des politiques publiques.

4. En particulier, sur la base de législation du régime général, disponible sur www.legislation.cnav.fr, qui retrace l'historique des règles successivement en vigueur. 
de mission au Conseil d'orientation des retraites $(\mathrm{COR})^{5}$. La première partie de l'article décrit le contexte économique dans lequel les changements législatifs et réglementaires sont intervenus depuis 1945 et la façon dont ces changements ont pu générer des contradictions entre les ambitions et les résultats. La deuxième partie examine dans quelle mesure le système actuel assure une solidarité en direction de ses assurés aux carrières discontinues, parfois à faibles salaires, et analyse les modalités d'acquisition et de valorisation des droits à retraite pour en pointer les défaillances. La troisième partie s'attache à identifier les freins au pilotage du système dans son fonctionnement même et discute, en particulier, de la capacité à l'adapter à un marché du travail en constante mutation.

\section{Un système confronté à la déstandardisation des carrières}

Le régime général de la Sécurité sociale est créé au sortir de la Seconde Guerre mondiale. Les salariés du commerce et de l'industrie sont alors affiliés de façon obligatoire aux Assurances sociales, créées en 1930, mais uniquement si leur rémunération ne dépasse pas un certain montant. Les fonctionnaires civils, les militaires et les salariés des grandes entreprises sont eux couverts par leurs régimes propres. En 1945, l'objectif est de créer un régime d'assurance retraite universel garantissant les moyens d'assurer sa subsistance à une population trop âgée pour subvenir à ses propres besoins par les fruits de son activité. La survie à des âges avancés est alors un « risque » que le système se charge de couvrir par les cotisations prélevées sur les actifs, selon le principe de la répartition. L'ambition d'un régime unique échoue devant les résistances des salariés du secteur public et des grandes entreprises publiques souhaitant conserver leurs régimes, et des non-salariés faisant valoir leurs spécificités professionnelles. Néanmoins, la couverture de l'assurance vieillesse des salariés du secteur privé s'étend. Ainsi, les règles du régime général sont reprises par les salariés agricoles (années 1950) et les artisans et commerçants (1973) au sein de régimes distincts. En 1975, la nécessité de justifier de quinze ans d'assurance pour ouvrir droit à une pension au régime général est supprimée. Les actifs ne satisfaisant pas la condition de durée minimale d'affiliation appliquée dans leur propre régime (celui de la fonction publique, par exemple) sont alors rattachés au régime général, notamment des femmes encore nombreuses à n'avoir que de courtes durées d'activité. Par ailleurs, la couverture se renforce : 1 'affiliation aux régimes complémentaires des non-cadres - $1^{\prime}$ Arrco $^{6}$ - et des cadres - l'Agirc $^{7}$ - devient obligatoire en 1972 et 1974 respectivement $^{8}$.

5. Les propos tenus dans cet article n'engagent en aucune façon ces deux institutions.

6. Association pour le régime de retraite complémentaire des salariés, créée en 1961.

7. Association générale des institutions de retraite complémentaire des cadres, créée en 1947.

8. Pour les agents non titulaires de la fonction publique, qui sont couverts en base par le régime général, l'affiliation se fait à l'Institution de retraite complémentaire des agents non titulaires de l'État et des collectivités publiques (Ircantec), créée en 1970. 
Le système de retraite des salariés du secteur privé se construit sur une base contributive liant le montant de la pension à l'effort contributif passé. À partir du début des années 1970, deux modes de fonctionnement coexistent : les annuités pour le régime général, régime de base, et les points pour les régimes complémentaires. Les pensions des régimes Arrco et Agirc sont calculées en valorisant les points acquis par les cotisations acquittées durant la période active. La pension du régime général correspond, elle, au produit d'un salaire de référence, calculé sur une partie de la carrière, et du taux de remplacement auquel s'engage le régime à un âge donné en contrepartie d'une certaine durée d'affiliation (encadré 1). Selon les périodes, les paramètres retenus dans la formule de calcul du régime général ont modifié les rôles respectifs de l'âge et du nombre d'annuités validées. Si, avant les années 1980, les conditions requises pour bénéficier du taux plein contraignaient à des départs que l'on peut juger tardifs au regard de l'espérance de vie en bonne santé, celles en vigueur dans les années 1980-1990 concentrent les départs à la retraite autour de 60 ans pour les hommes et de 65 ans pour les femmes. Ce cadre uniformisant s'applique pourtant à une population diverse et pour partie directement en prise avec les aléas du marché du travail. Sa remise en cause par les profondes évolutions socio-économiques amorcées durant les années 1960 conduit, dans les années 2000, à ouvrir la plage des âges possibles de départ à la retraite en contrepartie d'un renforcement du rôle de la durée d'assurance.

\section{ENCADRÉ 1}

\section{Les principes de calcul de la pension du régime général}

Deux bornes d'âge encadrent les conditions de départ à la retraite :

- l'âge à partir duquel peut être perçue la pension, passé de 60 à 62 ans en 2010 (hors mesures spécifiques) ;

- l'âge auquel est accordé le taux plein sans conditions de statut (inaptes, invalides) ou de durée d'assurance - c'est-à-dire le nombre d'annuités validées -, passé de 65 à 67 ans.

La pension versée par le régime général se calcule comme le produit de trois composantes :

- le salaire de référence ou « salaire annuel moyen », calculé sur les meilleurs salaires dans la limite du plafond de la Sécurité sociale (les dix derniers avant 1973);

- le coefficient de proratisation, qui rapporte la durée validée par l'assuré au régime général à la durée maximale rémunérée par le régime (37,5 années soit 150 trimestres jusqu'en 2003, puis variable selon la génération, 41,5 années soit 166 trimestres pour les générations 1955 à 1957 par exemple) ;

- le taux de liquidation, qualifié de « plein » à 50 \% (40 \% jusqu'en 1971) et « réduit » en deçà lorsqu'il lui est appliqué une décote.

La décote s'applique pour chaque trimestre manquant par rapport à l'âge automatique du taux plein ou, depuis le $1^{\text {er }}$ avril 1983, si c'est à l'avantage de l'assuré, par rapport à une durée cible. Cette durée est actuellement identique au nombre d'annuités prises en 
compte dans le calcul de la proratisation ; elle lui a été supérieure entre 1994 et 2007 selon les générations ${ }^{1}$.

Une surcote a par ailleurs été introduite en 2003, qui s'applique non sur le taux, mais sur le montant de la pension : elle valorise uniquement les trimestres cotisés au-delà de l'obtention du taux plein (par l'âge ou la durée).

$$
\text { Pension }=\text { salaire de référence } \times \frac{\text { durée validée au régime }}{\text { durée de proratisation }} \times \quad \begin{gathered}
\text { taux de liquidation } \\
\text { (âge de départ, durée validée tous régimes) }
\end{gathered}
$$

Le cœur du calcul de la pension, représenté par cette formule, est complété par deux grands types de dispositifs explicites de solidarité :

- les mesures de compensation des aléas de carrière et les droits familiaux consistent en l'octroi de trimestres qui s'ajoutent à la durée validée par cotisations et, pour certains d'entre eux (assurance vieillesse des parents au foyer, congé de maternité depuis 2012), le report de salaires sur le compte de l'assuré susceptibles d'entrer dans le calcul du salaire de référence ;

- le minimum contributif revient à relever le montant du salaire de référence à un niveau plancher, lorsque la pension est calculée à taux plein (par l'âge ou la durée), afin d'assurer une pension minimale.

1. Cette dissociation entre les deux paramètres de durée fait suite à la réforme de 1993. Celle-ci fait passer la durée requise pour le taux plein, sans toucher à la durée de proratisation, de 37,5 annuités pour la génération 1933 à 40 annuités à partir de la génération 1943, avec application à toutes les générations à partir de l'année 2003. La réforme de 2003 prévoit le réalignement des deux paramètres de durée et augmente, génération par génération, la durée de proratisation afin qu'elle atteigne 40 annuités pour la génération 1948 ; en 2008, cette durée est appliquée à toutes les générations. Les deux paramètres de durée augmentent ensuite simultanément, à un rythme générationnel, selon la règle d'évolution fixée par la réforme, en lien avec l'espérance de vie.

\section{L'édification d'un système solidaire, mais déstabilisant les fins de carrière}

Le contexte économique des années 1950-1960 dans lequel s'installe le régime général est propice au développement des mécanismes fidélisant la main-d'œuvre et valorisant l'ancienneté : la société salariale émerge (FouRCADE, 1992); la croissance soutenue s'accompagne d'une situation de quasi-plein-emploi avec un taux de chômage inférieur à $3 \%$; le recours croissant à des processus de production standardisés favorise une différenciation verticale des emplois et des compétences. Cependant, dès le début des années 1970, la durabilité des engagements en termes d'emploi et la valorisation de l'ancienneté sont remises en cause. L'accroissement de la concurrence internationale et la financiarisation réduisent l'horizon temporel des entreprises. Ces dernières externalisent les tâches qui dépassent leur cœur de métier et recherchent alors une main-d'œuvre plus polyvalente et plus flexible, aux compétences moins spécifiques et donc davantage transférables. Parallèlement, l'innovation technologique requiert de nouveaux savoirs et rend obsolètes les compétences accumulées (BEHAGHEL et al., 2010). De facto, les lignes hiérarchiques se raccourcissent et les sortants du 
système scolaire les plus diplômés accèdent directement aux postes les plus qualifiés, précédemment réservés aux travailleurs qui cumulaient plusieurs années d'ancienneté. Ces transformations organisationnelles et technologiques affectent principalement les travailleurs peu qualifiés et ceux jugés les moins capables de s'adapter. Les jeunes sont conduits à retarder leur entrée sur le marché du travail ; les plus âgés, dont les compétences spécifiques sont moins valorisables et qui réclament au contraire des investissements en formation risquant de ne pouvoir être amortis (AUBERT, CRÉPON, 2003), sont poussés vers le chômage ou la préretraite. Cette évolution est appuyée par un assouplissement de la réglementation du travail qui crée de nouvelles normes d'emploi. Les contrats à durée déterminée et le travail intérimaire deviennent les principales modalités d'entrée en emploi dès le début des années 1980. Le temps partiel se développe et accompagne l'essor de l'activité féminine. La période active se raccourcit et se brouille à ses deux extrémités (TROADEC, 1998 ; FOURNIER, GIVORD, 2001). L'emploi se concentre sur une phase comprise entre 25 et 50 ans, mais sans que sa stabilité soit assurée, car les transitions entre emploi, chômage et inactivité sont plus fréquentes et concernent un nombre croissant de personnes (AMOsSÉ, 2002 ; CoE, 2009).

Pour prévenir des ruptures d'acquisition de durée d'assurance en cas d'interruption d'activité, plusieurs dispositifs explicites de solidarité sont introduits durant les années 1970. Ainsi, au régime général, des trimestres sont accordés sans contrepartie de cotisations en cas de chômage, de maladie et de service militaire. Afin d'améliorer la situation des femmes et leur faciliter la constitution de droits propres à pension malgré les charges familiales qui pénalisent souvent leur activité professionnelle, les droits familiaux sont développés : en 1972, des trimestres d'assurance sont accordés aux mères pour chaque enfant élevé et une assurance vieillesse des mères de famille est créée, transformée en 1979 en assurance vieillesse des parents au foyer (AVPF), qui attribue des droits à retraite sur la base d'une activité rémunérée au salaire minimum aux prestataires de certaines allocations familiales (BROCAS, 2004 ; BAC, 2011). Les régimes complémentaires adoptent des mesures similaires, sur un périmètre plus étroit cependant ${ }^{9}$. À partir de 1983, le « minimum contributif » assure une pension minimale aux assurés du régime général ayant eu de faibles salaires et faisant valoir leurs droits à retraite à taux plein au terme d'une carrière complète ou à 65 ans.

Dès 1971, les discussions autour de la loi Boulin engagent le débat sur la baisse de l'âge légal de départ à la retraite. La pension représente alors $40 \%$ du salaire de référence à 65 ans. La pension peut être demandée dès 60 ans, mais les assurés subissent une décote de $4 \%$ sur ce taux (et donc du salaire de référence) par année d'anticipation, réduisant de fait leur pension de moitié. Durant les années 1970, l'accès au taux plein à 60 ans est accordé par dérogation à des populations spécifiques, comme les personnes reconnues inaptes (1971) ou les mères de famille ouvrières (1975),

9. Pour un panorama de ces droits, voir par exemple les séances du COR des 25 mai 2011 et 9 juillet 2014. Les documents sont disponibles sur le site du Conseil : http://www.cor-retraites.fr/article389.html et http://www.cor-retraites. fr/article439.html, consultés le 30 juin 2017. 
puis ouvert à toutes les femmes (1979) (BISSERET, 1979). L'ordonnance du 26 mars 1982 finalise la généralisation en l'accordant également aux hommes. L'âge minimal de liquidation reste inchangé, 60 ans, mais les assurés ne subissent plus de pénalités financières au régime général et dans les régimes complémentaires s'ils justifient d'une durée d'assurance suffisante ${ }^{10}$. En 1982, cet abaissement de l'âge de la retraite à taux plein institutionnalise une période de non-travail après la vie active en la rendant accessible au plus grand nombre. En effet, alors qu'à la création du régime, l'espérance de vie à la naissance était de 60 ans pour les hommes, 65 ans pour les femmes en France métropolitaine, vingt-cinq ans après, plus de dix années d'espérance de vie ont été gagnées par les hommes, près de quinze par les femmes. La réforme de 1982 est présentée comme offrant la garantie d'un droit durable au repos à des travailleurs sans emploi en fin de carrière du fait de la crise économique, et de nature à améliorer le ratio entre la durée de retraite et la durée d'activité pour les ouvriers et les employés. Elle vise explicitement à décourager la poursuite d'activité à des âges avancés ; la surcote qui valorisait les départs après 65 ans est ainsi supprimée. Les paramètres de calcul alors fixés incitent les assurés à faire valoir leurs droits à retraite dès l'obtention du taux plein : les assurés aux carrières les plus courtes reculent leur départ en retraite jusqu'à justifier des conditions requises en termes d'âge ou de durée d'assurance, et les assurés aux carrières les plus longues sont découragés de différer la demande de leur pension (BLANCHET, MAHIEU, 2004). La durée d'assurance requise pour le taux plein, qui est alors de 37,5 années, correspond à une carrière continue entamée à l'âge de 22-23 ans. Les femmes, dont les carrières sont parfois marquées par de longues périodes d'inactivité, sont nombreuses à attendre l'âge de 65 ans pour partir à la retraite. En bénéficiant du taux plein, elles accèdent aussi au minimum contributif si leurs salaires ont été faibles. La majorité des hommes peut prétendre au taux plein dès 60 ans, car leur entrée dans la vie professionnelle s'est effectuée dans un contexte économique favorable, souvent précocement.

La réforme de 1982 intervient dans un contexte où les cessations d'activité avant la retraite se multiplient, sont encouragées par l'État et, pour certaines, financées sur fonds publics (allocations spéciales du Fonds national de l'emploi, par exemple). Elles apparaissent comme un moyen de lutter contre le chômage en libérant des emplois, et concilient l'intérêt des travailleurs, qui bénéficient de façon anticipée d'un droit à l'« inactivité pensionnée » (GuILLEMARD, 1993, p. 276), avec l'intérêt des employeurs, qui se séparent de travailleurs potentiellement coûteux. La générosité des dispositifs de cessation précoce d'activité et les incitations financières du système de retraite pour liquider la pension à taux plein ont néanmoins pour effet de rétrécir les perspectives d'emploi des travailleurs les plus âgés (AUBERT, 2012 ; COURTIOUX, ERHEL, 2005). En fin de compte, la politique de gestion de l'emploi par les âges se solde par un double échec. D'une part, le taux d'emploi des 50-64 ans passe de $57 \%$ à $44 \%$ entre 1980 et 1995, mais les gains attendus du partage de l'emploi au profit des jeunes ne se

10. Dans le même temps, les abattements dans les régimes complémentaires qui s'appliquaient avant 65 ans sont supprimés pour les assurés justifiant du taux plein au régime général. 
produisent pas ; le taux de chômage des $15-24$ ans passe de $12 \%$ à $20 \%$ sur la même période. D'autre part, la situation financière du système de retraite est grevée par les pensions versées dès 60 ans à des personnes qui auraient pu souhaiter continuer de travailler et cotiser.

\section{À la recherche d'un équilibre entre viabilité financière et justice sociale}

Au début des années 1990, l'objectif de parité de niveau de vie entre actifs et retraités est quasiment atteint ${ }^{11}$ et le débat sur la redistribution se centre sur la question du partage du revenu entre actifs et inactifs âgés (BROCAS et al., 1992). Avec l'accroissement continu des dépenses de retraite lié à l'arrivée à maturité des régimes et le vieillissement des premières générations du baby-boom qui arrivent en fin de carrière, la question de la viabilité financière du système se pose alors. Elle entre véritablement dans le débat public avec deux rapports du Commissariat au Plan : le Livre blanc sur les retraites en 1991 et le rapport Charpin en 1999.

Ce nouvel impératif financier prend corps avec la réforme de 1993 qui renforce la contributivité, tant au niveau individuel qu'au niveau du financement du système. En effet, pour la première fois, les paramètres de la formule de calcul de la pension du régime général sont durcis. La montée en charge est progressive jusqu'en 2008, suivant un rythme générationnel : la durée d'assurance requise pour bénéficier d'une pension à taux plein passe de 37,5 à 40 annuités, le nombre de salaires retenus pour le calcul du salaire de référence augmente des 10 aux 25 meilleures années d'activité. En outre, la réforme confirme l'indexation sur les prix pratiquée depuis 1987 des pensions et des salaires passés considérés pour le calcul du salaire de référence. Lors du départ à la retraite, les salaires portés au compte des assurés sont ainsi actualisés sur la base de l'inflation (hors tabac) et non plus sur la croissance du salaire moyen par tête, généralement plus dynamique ${ }^{12}$, ce qui s'avère d'autant plus défavorable que le nombre de salaires retenus est important ${ }^{13}$. Par ailleurs, la réforme externalise le financement de certains dispositifs de solidarité du régime général en les faisant prendre en charge par le Fonds de solidarité vieillesse (FSV) nouvellement créé. La contribution sociale généralisée (CSG), les impôts et taxes affectés qui l'alimentent complètent, ou se substituent, alors aux cotisations pour financer les droits accordés. Le

11. En 1970, le revenu médian par unité de consommation des ménages retraités était de $40 \%$ inférieur à celui des actifs ; en 1990, l'écart s'est réduit à $16 \%$. Alors que le revenu des actifs n'a augmenté que de $8 \%$ sur la décennie 1980, celui des retraités a crû de $40 \%$ dans le même temps (HourriEZ, RouX, 2001).

12. La croissance du pouvoir d'achat des salaires a cependant été relativement modérée durant les années 1980 et au début des années 1990. Entre 1993 et 1996, le taux de croissance du salaire moyen est même inférieur à celui des prix. La désindexation a alors pu être perçue par certains comme le moyen de protéger les retraités des aléas de la conjoncture. 13. Si les prix augmentent moins vite que les salaires, l'actualisation sur les prix conduit à moins valoriser les salaires passés, et ce d'autant plus qu'ils sont éloignés du départ à la retraite. Cela se traduit par une dégradation du salaire de référence qui est d'autant plus importante que le nombre de salaires retenus pour son calcul est élevé et que sont pris en compte des salaires de début de carrière. L'une des conséquences de la revalorisation sur les prix des salaires passés est qu'au terme d'une carrière complète rémunérée au plafond de la Sécurité sociale, le taux de remplacement du dernier salaire est inférieur à $50 \%$ du plafond qui, lui, évolue comme le salaire moyen par tête. 
champ couvert par le FSV, limité initialement aux périodes validées au titre du chômage et aux majorations pour enfants et conjoint à charge, sera ensuite continûment élargi.

Dix ans après la réforme de 1993, celle de 2003 s'inscrit dans un contexte où, sous l'impulsion des institutions européennes (JOLIVET, 2002), la « culture du départ précoce » laisse place au « vieillissement actif », c'est-à-dire à une situation où la norme - le droit et le devoir - est de rester sur le marché du travail. La prolongation d'activité est alors vue comme un facteur de croissance économique. La réforme de 2003 renforce la centralité de la durée d'assurance dans le calcul de la retraite du régime général, mais dans un cadre liant davantage les évolutions du système avec l'environnement démographique et économique. Elle met en place les conditions d'un prolongement des carrières au-delà de 60 ans (recentrage des cessations précoces d'activité sur les emplois à forte pénibilité, libéralisation du cumul emploi-retraite, etc.) et instaure un mécanisme d'ajustement semi-automatique de la durée requise sur les gains d'espérance de vie : les paramètres de durée sont calculés lors de rendez-vous quadriennaux et réajustés, si nécessaire, en fonction de l'évolution de l'emploi, des taux d'activité des plus de 50 ans et de la situation financière des régimes. En contrepartie, la réforme ouvre le départ à la retraite dès 56 ans aux personnes aux longues carrières, justifiant d'une entrée précoce dans la vie active et d'une longue durée de cotisation ${ }^{14}$. Une surcote valorisant les départs à la retraite au-delà du taux plein est réintroduite et la décote est réduite afin d'alléger les pénalités des assurés qui feraient valoir leurs droits précocement, par choix ou nécessité. Ce nouveau jeu de paramètres contraint moins les départs à la retraite vers le taux plein (BRIARD, MAHFOUZ, 2011) et participe certainement à la remontée des taux d'emploi des 55-64 ans de plus de 12 points sur la décennie, rattrapant ainsi le niveau du début des années $1980^{15}$. La réforme vise aussi à mieux prendre en compte les débuts de carrière en ouvrant la possibilité de « racheter» des trimestres pour les années d'études supérieures.

La crise économique et financière de la fin des années 2000 dégrade fortement les ressources du système de retraite ; les besoins de financement prévus avant crise pour 2025 sont alors déjà atteints (CoR, 2010b). Pour y répondre, la réforme de 2010 met fin à la retraite à 60 ans : les bornes d'âges sont relevées de deux ans, de 60 à 62 ans pour l'âge légal, de 65 à 67 ans pour l'âge d'annulation de la décote. Hors situation de handicap et de pénibilité, les départs à l'âge de 60 ans ne restent possibles que dans le cadre du dispositif « longues carrières ». Ce dispositif est réformé en 2012 pour le maintenir ouvert à des générations soumises à l'obligation de scolarité jusqu'à 16 ans et à des durées requises plus longues ${ }^{16}$. La réforme de 2014 acte par ailleurs le principe

\footnotetext{
14. La mesure a connu un succès inattendu les premières années, en raison du nombre important d'assurés régularisant des cotisations arriérées pour remplir les critères d'éligibilité stricts d'âge de début d'activité.

15. Le taux d'emploi « sous-jacent », corrigé des effets de composition démographique, passe ainsi de $34 \%$ en 2003 à plus de $40 \%$ à partir de 2010. Il est de $50 \%$ en 2014.

16. Avant le décret du 2 juillet 2012, les conditions d'éligibilité au dispositif « longues carrières » sont triples : justifier d'une durée totale d'assurance supérieure d'au moins huit trimestres à celle requise pour le taux plein ; justifier d'une condition de durée cotisée correspondant à la durée pour le taux plein pour un départ à 59 ans, majorée de quatre trimestres pour un départ à 58 ans, de huit trimestres pour un départ à 56 ou 57 ans ; justifier de quatre ou cinq trimestres
} 
d'un allongement de la durée d'assurance au fil des générations en figeant le calendrier de montée en charge, supprimant les clauses de revoyure quadriennales instaurées en 2003. Par ailleurs, elle complète les dispositifs de compensation de droits en élargissant ceux liés à la maternité, au chômage non indemnisé et aux périodes d'études et de formation. Elle baisse aussi le salaire seuil validant un trimestre ( 150 heures de salaire minimum interprofessionnel de croissance - Smic - au lieu de 200 heures) pour rendre moins pénalisant le temps partiel, ainsi que les situations de multiactivité ou de mobilité entre régimes de retraite. Elle crée en outre un compte personnel de prévention de la pénibilité ouvrant droit à un départ précoce en cas d'exposition à des facteurs de pénibilité durant la carrière. Elle apporte ainsi une réponse, au moins partielle, à des revendications de longue date pour prendre en compte les conditions de travail dans la retraite des salariés du secteur privé ${ }^{17}$.

Les années 2000-2010 témoignent d'une modification profonde de la façon de penser le système de retraite dans son environnement. L'échec de la politique de gestion de l'emploi par les âges menée durant les années 1980 a contredit l'idée d'une homogénéité des situations individuelles que le système aurait pu traiter équitablement de façon uniforme. Durant la décennie 1990, le recentrage sur la question de sa viabilité financière a conduit à renforcer le lien entre emploi et retraite dans la détermination des droits à pension, les dispositifs explicites de compensation des aléas restant inchangés. Ce n'est que dix ans plus tard, à partir de 2003 et de la mise en place de plusieurs dispositions spécifiques, que le socle d'un nouveau paradigme pour le système s'affirme, celui d'une logique de contributivité renforcée conférant un rôle central à la durée d' assurance, adossée à une bonne couverture des risques de carrière. Les réformes et révisions ponctuelles des années 2010 vont confirmer cette évolution et améliorer la couverture des risques déjà reconnus et l'étendre à de nouveaux. En somme, il s'agit de mieux prendre en compte la diversité des situations et des choix individuels, en particulier les écarts par rapport au standard de l'emploi continu à temps complet jusqu'à 60 ans, de façon à rendre le système de retraite plus en phase avec les évolutions démographiques et socio-économiques, tout en le maintenant sur une trajectoire financièrement soutenable.

\footnotetext{
d'assurance avant la fin de l'année civile où est survenu le $16^{\mathrm{e}}$ ou $17^{\mathrm{e}}$ anniversaire selon l'âge de départ. Le décret du 2 juillet 2012 relève la borne d'âge de début d'activité à 20 ans pour les départs à 60 ans, supprime la condition de durée totale et assouplit la notion de trimestres réputés cotisés. Pour une présentation de la mesure, voir le document II-09 de la séance du COR du 25 septembre 2012, en ligne : http://www.cor-retraites.fr/IMG/pdf/doc-1842.pdf, consulté le 30 juin 2017.

17. Depuis le début du $\mathrm{XIX}^{\mathrm{e}}$ siècle, la fonction publique permet aux catégories dites « actives », occupant certains emplois présentant un « risque particulier » ou des « fatigues exceptionnelles », de partir à la retraite précocement (cinq à dix ans avant l'âge légal selon les cas). Des mesures similaires de départs anticipés sont appliquées par plusieurs régimes spéciaux (SNCF, RATP, industries électriques et gazières, etc.).
} 


\section{Des règles de calcul aux effets incertains sur les carrières non standards}

Les effets croisés du système de retraite et de l'évolution des situations sur le marché du travail rendent d'autant plus nécessaire, mais d'autant plus complexe, le calibrage des paramètres du système au regard des objectifs que la loi lui assigne. Celle-ci affirme le caractère contributif du système de retraite, qui « assure aux retraités le versement de pensions en rapport avec les revenus qu'ils ont tirés de leur activité ». Elle prévoit également pour les assurés « un traitement équitable au regard de la durée de la retraite comme du montant de leur pension, quels que soient leur sexe, leurs activités et parcours professionnels passés, leur espérance de vie en bonne santé, les régimes dont ils relèvent et la génération à laquelle ils appartiennent ». Elle pose enfin « un objectif de solidarité entre les générations et au sein de chaque génération, notamment par l'égalité entre les femmes et les hommes, par la prise en compte des périodes éventuelles de privation involontaire d'emploi, totale ou partielle, et par la garantie d'un niveau de vie satisfaisant pour tous les retraités ${ }^{18} »$.

Le seul objectif d'équité intragénérationnelle doit ainsi s'exercer dans de multiples dimensions. Les objectifs d'équité entre les femmes et les hommes, entre régimes de retraite ou selon l'état de santé interrogent de façon large sur les inégalités de conditions de déroulement des carrières, dues notamment à une asymétrie de la charge familiale, à des rapports spécifiques entre salariés et employeurs, ou à des situations professionnelles pénibles ou dangereuses ${ }^{19}$. L'objectif de traitement équitable selon les parcours professionnels, auquel peut se rattacher la solidarité à l'égard des travailleurs aux carrières involontairement discontinues, a plus directement trait aux carrières ellesmêmes, indépendamment des caractéristiques des assurés concernés ${ }^{20}$. À cet égard, l'équité au regard des parcours professionnels peut être vue comme une dimension cardinale de l'équité en matière de retraite, selon laquelle une redistribution des assurés aux capacités contributives les plus importantes, dont les carrières sont longues et les salaires élevés, doit s'exercer en direction des assurés aux carrières plus courtes et/ou à salaires plus faibles. Il ne s'agit pas d'atteindre une égalité de situations en termes de montant et/ou d'âge de la retraite, mais de prévenir, au moins pour partie, certains effets négatifs de situations jugées comme devant relever de la solidarité, telles que le chômage ou la maladie.

\footnotetext{
18. II de l'article L111-2-1 du Code de la Sécurité sociale.

19. Sur ces questions, nous renvoyons aux réflexions menées par le COR (CoR, 2013 : chapitre 5) et, plus spécifiquement, aux travaux sur les droits familiaux et conjugaux de retraite (COR, 2008), sur les différences de retraites entre salariés du secteur privé et fonctionnaires (CoR, 2015) et sur la prise en compte de la pénibilité et de l'état de santé (COR, séance du 23 novembre 2016, en ligne : http://www.cor-retraites.fr/article478.html, consultée le 30 juin 2017). 20. Par exemple, l'équité au regard des parcours peut conduire à s'interroger sur les droits à retraite accordés au titre des périodes de maladie, mais sans prendre en considération la pénibilité des activités exercées par les travailleurs.
} 
Au sein du système de retraite, cette mission redistributive est assignée aux régimes de base. Elle s'est affirmée durant la décennie 1970, mais le diagnostic sur l'effectivité de la redistribution globale du système et, plus encore, la compréhension des mécanismes sous-jacents sont relativement récents, le jeu complexe des interactions entre les caractéristiques de carrière et les modalités de calcul du régime général les ayant rendus difficiles (encadré 2).

\section{ENCADRÉ 2}

\section{L'évaluation de l'équité intragénérationnelle en matière de retraite, une histoire récente}

Au sein du système de protection sociale, l'assurance vieillesse a pour particularité de faire de l'assuré successivement un financeur et un prestataire. La solidarité intragénérationnelle ou la redistribution en matière de retraite doit donc s'apprécier sur l'ensemble du cycle de vie, ce qui suppose de connaître la chronique des contributions et des prestations, autrement dit de suivre les carrières dans leur intégralité en parallèle des changements législatifs.

Le diagnostic sur la redistributivité du système est longtemps resté partiel par manque d'outils adéquats d'évaluation et de prospective, qui doivent reposer sur des données suffisamment détaillées et complètes pour retracer les droits à retraite en cours de constitution des assurés en activité. En l'absence de telles données, des évaluations peuvent toutefois être réalisées à partir de cas types de carrière (voir par exemple, El MeKKAOUI De FreITAS et al., 2011). La méthode permet de dissocier les effets propres à la législation de ceux liés aux caractéristiques individuelles, lesquelles sont parfaitement contrôlées soit parce qu'elles sont théoriques, soit parce qu'elles sont calées sur des moyennes. Cependant, l'approche reste analytique, car elle ne permet pas d'inférer des effets à un niveau agrégé, faute de pouvoir apprécier la représentativité des cas types dans la population ni de pouvoir couvrir la diversité des situations individuelles.

Les premiers exercices d'évaluation du caractère redistributif global du système de retraite ont reconstitué des cohortes fictives à partir de données en coupe segmentant la population par catégories sociales (LAGARDE, Worms, 1978). Durant les années 1980, la mise en place de panels ouvre la voie au développement des analyses longitudinales. À partir du panel des Déclarations annuelles de données sociales (DADS), Laurent CAUSSAT (1996) étudie ainsi les mécanismes de redistribution de la formule de calcul des pensions sur les carrières salariales pour partie projetées des salariés de la génération 1950. Il met en évidence des effets redistributifs ambigus entre les catégories sociales, qui suggèrent l'existence d'effets antiredistributifs implicites dans les règles de calcul, faisant que, par exemple, relativement aux cotisations versées, des personnes ayant eu des salaires élevés peuvent avoir de meilleures pensions que celles ayant eu des salaires plus faibles. Il conclut à la nécessité d'analyser les mécanismes redistributifs en différenciant la population assurée par les caractéristiques des carrières plutôt que par grandes catégories sociodémographiques qui agrègent des situations individuelles hétérogènes. Les travaux qui suivent délaissent de fait l'analyse par catégories sociales, privilégiant une différenciation par profils de carrière (BRIARD, LELIEUR, 2007) ou par niveau de rémunération, qui est 
calculé soit sur l'ensemble de la carrière - en intégrant donc la durée de la carrière - soit à partir de salaires représentatifs d'un niveau moyen ou de fin de carrière (voir par exemple Aubert, BACHELET, 2012).

Ces travaux évaluent souvent la redistribution à partir de plusieurs types d'indicateurs, chacun présentant des limites. Les écarts entre les différentes catégories de population étudiées (catégories sociales ou profils de carrière, par exemple) sur lesquelles ces indicateurs sont calculés renseignent alors sur le sens et l'ampleur de la redistribution entre ces catégories. Certains de ces indicateurs prennent en compte l'ensemble des revenus sur le cycle de vie. Ainsi, le taux de récupération, qui rapporte les flux actualisés de cotisations aux flux actualisés de pensions, équivaut à un taux de rentabilité de l'opération retraite indiquant le montant de pension servi par euro de cotisation. Il suppose néanmoins de choisir un taux d'actualisation pertinent. Parce qu'il permet de s'affranchir de cette difficulté, le taux de rendement interne, qui correspond au taux d'actualisation qui assure l'égalité entre les flux actualisés de cotisations, est plus largement utilisé. Ces approches comptables ont cependant l'inconvénient de considérer de façon équivalente le fait de percevoir une faible pension sur une longue période de retraite et une pension élevée sur une courte période. Il leur est donc parfois préféré l'indicateur plus familier du taux de remplacement, qui rapporte la pension à la masse des salaires passés ou au salaire moyen de carrière, ou le taux d'annuité, qui rapporte le taux de remplacement du salaire moyen à la durée de carrière et s'interprète comme la part du salaire moyen versée sous forme de rente pour chaque année validée. Pour tenir compte de la durée de versement des pensions, un taux de prestations peut aussi être calculé, en multipliant le taux d'annuité par la durée de versement. Ces indicateurs sont des mesures relatives des pensions par rapport aux salaires. Des indicateurs sur le niveau et la dispersion du montant des pensions sont donc également nécessaires pour apprécier plus globalement l'étendue de la redistribution et l'adéquation du système à son objectif d'assurer un niveau de retraite suffisant.

\section{Des règles de calcul complexes aux effets non maîtrisés}

Dans le système de retraite public français, la redistribution peut être assimilée à l'absence de contributivité stricte. Au sens strict, la contributivité établit une proportionnalité entre cotisations et pensions, indifférenciée selon les travailleurs : chacun reçoit en fonction de ce qu'il a cotisé dans les mêmes proportions. À l'opposé, la redistribution s'opère lorsque la relation entre les revenus et les pensions diffère entre les travailleurs, dans le sens où ceux dont les revenus professionnels ont été élevés reçoivent proportionnellement moins en termes de pensions que ceux dont les revenus ont été plus faibles. En raison de la faible différenciation des taux de cotisation selon les revenus, la redistribution a donc lieu dès lors que ceux dont l'effort contributif est le plus important - du fait de leur durée de carrière et/ou de leurs salaires, relativement élevés - reçoivent proportionnellement moins que ceux dont l'effort contributif a été plus faible.

Les non-linéarités de la formule de calcul de la pension du régime général engendrent de fait des transferts entre les assurés, qui peuvent s'avérer redistributifs ou antiredistributifs, en étant, dans ce dernier cas, à la défaveur des assurés aux carrières 
courtes et/ou aux salaires modestes. Une première source de non-proportionnalité entre cotisations et pensions est due à la relation complexe entre l'âge de départ à la retraite et la durée d'assurance, les écarts entre l'âge effectif de départ du travailleur et les âges légaux de départ n'étant pas valorisés de la même façon que les écarts entre sa durée d'assurance validée et le nombre d'annuités requises pour le taux plein ou la proratisation. Selon les périodes, les paramètres retenus dans la formule ont généré des distorsions plus ou moins importantes entre âge et durée, tout en faisant jouer un rôle croissant à la durée d'assurance par rapport à l'âge (BRIARD, MAHFOUZ, 2011). Les règles actuelles produisent des effets redistributifs ambigus selon la durée passée en emploi (AUBERT, 2015). Elles bénéficient principalement aux assurés atteignant la durée requise pour le taux plein exactement à l'âge d'ouverture des droits, mais aussi aux assurés ayant les carrières les plus courtes, sortis des études très jeunes ou ayant les salaires les plus faibles qui, en pratique, sont les premiers à bénéficier des dispositifs explicites de compensation de la durée d'assurance.

Une autre source de non-proportionnalité est la non-séparabilité complète des termes relatifs aux salaires et à la durée dans la formule de calcul. En effet, le salaire de référence - ou «salaire annuel moyen »- est calculé non sur la totalité de la durée ayant donné lieu à cotisations, mais seulement sur une partie et les trimestres d'assurance sont validés, non en fonction de la durée d'activité, mais par rapport à un niveau de salaire (depuis 2014, 150 heures payées au salaire minimum valident un trimestre). Or, dans les faits, les durées d'assurance validées et les rémunérations sont liées de façon non monotone : par exemple, parmi les personnes nées en 1946, celles ayant eu les salaires les plus élevés sont en proportion croissante jusqu'à une durée d'assurance d'environ quarante ans. Au-delà, la relation s'inverse en raison du nombre relativement plus important de personnes peu diplômées ayant commencé à travailler jeunes. Ainsi, les personnes dont le salaire de carrière est parmi les $25 \%$ plus élevés sont en proportion décroissante parmi celles dont la durée est supérieure à 42 ans pour les femmes, 38 ans pour les hommes, ces durées étant de l'ordre de 43 et 41 ans respectivement si l'on considère les personnes rémunérées au-dessus du salaire médian (AUBERT, Duc, 2011). La non-séparabilité des composantes salariale et de durée dans la formule de calcul en annuités rend donc difficilement maîtrisables les effets combinés des règles de calcul. Par exemple, en pratique, la sélection des meilleures années dans le salaire de référence conjuguée au plafond de la Sécurité sociale, lequel détermine les parts respectives des pensions de base et complémentaires et donc les redistributions associées, accentue les inégalités de carrière (AUBERT, BACHELET, 2012).

\section{Un système globalement compensateur, malgré des inefficacités}

Malgré des effets antiredistributifs au cœur de la formule de calcul du régime général, les évaluations récentes concluent au caractère globalement redistributif du système, grâce en particulier aux dispositifs explicites de solidarité (CoR, 2010a, 2013). 
Un indice de l'effet redistributif du système est le resserrement de la distribution des pensions par rapport à celle des salaires cumulés sur toute la carrière. Pour les retraités des secteurs privé et public nés entre 1955 et 1964, le rapport entre les pensions des $10 \%$ les mieux lotis et des $10 \%$ les moins bien lotis est de 4, alors que le rapport en termes de salaires cumulés, qui dépend à la fois de la durée de la carrière et du niveau des rémunérations, est de 6,5 (AUBERT, BACHELET, 2012). La réduction des inégalités de carrière est encore plus importante pour les femmes, le ratio passant de 8,5 à 5,5. Une évaluation plus complète des transferts entre les assurés, tenant compte de la durée de versement des pensions, confirme l'effectivité de la redistribution du système, du régime général en particulier, en direction des assurés aux carrières les plus courtes et/ou les moins rémunérées (BRIARD, LELIEUR, 2007 ; WALRAET, VinCENT, 2003). Les femmes sont les premières destinataires de cette redistribution et cela indépendamment de leur espérance de vie plus longue que les hommes : grâce aux droits familiaux dont elles sont les principales bénéficiaires, le taux de rendement de la retraite ${ }^{21}$ des femmes dont la carrière est très courte et s'interrompt avant l'âge de 30 ans est d'un tiers supérieur à celui des hommes aux carrières similaires; le taux de rendement des femmes aux carrières interrompues entre 25 et 35-40 ans et aux salaires inférieurs au Smic est lui supérieur de l'ordre de $40 \%$ à celui des hommes (BRIARD, LELIEUR, 2007).

Les dispositifs visant explicitement à corriger les aléas de carrière (pension minimum, prise en compte du chômage, de la maladie, etc.) et les droits familiaux sont les principaux mécanismes qui contribuent à une redistribution en direction des assurés aux carrières peu rémunérées et/ou discontinues. En effet, la pension minimum permet aux assurés ayant eu de faibles rémunérations et une carrière complète de bénéficier d'une pension correspondant à celle perçue en théorie au terme d'une carrière continue au $\mathrm{Smic}^{22}$. Les trimestres d'assurance accordés en cas d'interruption d'activité, pour cause de chômage ou de maladie par exemple ${ }^{23}$, corrigent relativement bien les discontinuités de carrière en termes de durée dans les cas les plus fréquents (Colin, METTE, 2003 ; El Mekkaoui De Freitas et al., 2011). Par ailleurs, ils assurent indirectement une redistribution verticale, car les assurés pour lesquels ces trimestres sont utiles pour compléter la durée d'assurance ont aussi plus fréquemment des salaires faibles.

Toutefois, la capacité de ces dispositifs à assurer une couverture équitable pose question, car pour certaines configurations de carrière, leur effet est limité par les modalités de prise en compte et de valorisation de la durée d'assurance et par les règles de calcul du salaire de référence. En effet, la validation d'un trimestre supplémentaire peut ne pas augmenter la durée d'assurance si quatre trimestres sont déjà validés dans l'année où se produit l'aléa. Dans le cas où le trimestre augmente effectivement la

21. Il s'agit du taux de rendement interne ; voir encadré 2 . Les majorations de pension pour enfants ne sont pas incluses. 22. L'indexation des salaires étant réalisée sur la base de l'inflation et non de la croissance des salaires, le taux de remplacement net à la liquidation des retraites de base et complémentaires est un peu inférieur aux $85 \%$ du Smic net fixés par la réforme de 2003 (82\% pour la génération 1954 selon le rapport annuel du COR de juin 2016).

23. Un trimestre est accordé dès le $50^{\mathrm{e}}$ jour de chômage indemnisé ou non indemnisé sous conditions, et dès le $60^{\mathrm{e}}$ jour de maladie indemnisée. 
durée, l'effet sur la pension dépend des conditions remplies par l'assuré à son départ à la retraite. La valeur du trimestre peut par exemple être nulle si le départ à la retraite intervient à l'âge minimum légal et que la durée d'assurance validée est déjà suffisante pour prétendre à une retraite à taux plein. Dans le cas contraire, elle dépend du reste de la carrière, y compris des périodes relevant d'autres régimes puisque c'est la durée tous régimes confondus qui détermine le taux de la pension. Par ailleurs, les dispositifs de compensation de durée n'assurent pas, par nature, de compensation sous forme de salaires ${ }^{24}$. Les modalités de calcul du salaire de référence peuvent alors être préjudiciables, avec des effets très variables selon le moment où intervient l'interruption de carrière salariale. En effet, si la sélection des meilleurs salaires dans le salaire de référence permet d'exclure les salaires les moins élevés du calcul de la pension, elle bénéficie peu aux personnes ayant autour de vingt-cinq années travaillées, plus encore, si elles ont des salaires fluctuants ou ont leur pension relevée au minimum contributif en raison de faibles salaires (AUBERT, Duc, 2011). De plus, le calcul sur la base de salaires annuels, sans lien avec la durée effectivement travaillée dans l'année, est plutôt défavorable aux personnes ayant de courtes périodes d'emploi dans l'année, quelle qu'en soit la cause (chômage, emplois saisonniers, etc.). Les personnes polyaffiliées, dont certaines périodes d'activité relèvent d'autres régimes de base que le régime général, sont donc particulièrement pénalisées (CoR, 2011).

Les articulations complexes des règles de calcul des pensions produisent donc des inégalités au cœur même de la redistribution assurée par le système. L'augmentation du nombre de salaires pris en compte dans le salaire de référence suite à la réforme de 1993 a accentué ces mécanismes, mais peut-être davantage en raison du maintien de la règle d'un nombre fixe de salaires que par le renforcement de la contributivité induit par l'augmentation de ce nombre. Sans remettre en cause le fonctionnement en annuités, rendre le système plus juste au regard des parcours professionnels suppose de neutraliser les antiredistributions implicites. Un schéma de réforme serait ainsi de renforcer le caractère contributif du cœur du système, mais de façon à conserver la maîtrise des effets des dispositifs redistributifs ${ }^{25}$. Une solution consisterait à calculer un salaire de référence sur l'ensemble de la carrière, éventuellement en ôtant les salaires les plus faibles, tout en procédant à un relèvement du taux de liquidation et à une redéfinition des dispositifs explicites de compensation des interruptions d'activité. Pour les situations jugées devoir être couvertes par la solidarité, une option serait par exemple d'associer un équivalent salaire à chaque validation de trimestre ; ce salaire qui serait pris en compte dans le salaire de référence pourrait être fixé forfaitairement ou défini par rapport aux salaires précédemment perçus ${ }^{26}$. L'évolution récente des

\footnotetext{
24. Le congé de maternité est une exception depuis 2012, car les indemnités journalières entrent dans le calcul du salaire de référence.

25. Par exemple, en simulant un fonctionnement en comptes notionnels du régime général - schématiquement, un régime en points dont la valeur de service augmente avec l'âge de départ et diminue avec l'espérance de vie de la génération -, Christophe AlberT et Jean-Baptiste OliveAu (2011) montrent qu'un renforcement de la contributivité n'est pas incompatible, à coût constant, avec une réduction des inégalités de pension.

26. La validation de points gratuits dans les régimes complémentaires repose sur un principe similaire.
} 
dispositifs explicites de solidarité semble d'ailleurs confirmer cette orientation : les indemnités journalières du congé de maternité, majorées de $25 \%$, sont susceptibles d'être retenues dans le salaire de référence depuis 2012, et l'assurance vieillesse des parents au foyer, qui consiste en le report sur le compte de l'assuré des salaires basés sur le Smic, a été étendue au congé de proche aidant sans considération des ressources par la réforme de 2014.

\section{Un ajustement du système contraint par l'inertie et l'incertitude}

La politique de retraite s'inscrit sur un temps long, mettant à distance la prise de mesures et les effets produits. Ainsi, les inefficacités du système dans la gestion des fins de carrière et la prise en compte des carrières non standards n'ont été mises en évidence que lorsque certaines des personnes concernées étaient déjà parties à la retraite. Malgré le développement de l'évaluation prospective, l'incertitude temporelle reste une contrainte majeure pour définir la politique de retraite et piloter le système de retraite au regard de ses différents objectifs. Au cours des dernières années, les travaux d'analyse ont toutefois permis de mieux comprendre les mécanismes complexes des règles de calcul et d'ajuster le système de façon à assurer un traitement plus équitable des assurés.

\section{L'évaluation à l'épreuve du temps long et de la complexité}

La perspective de long terme qui caractérise la politique de retraite s'accompagne d'incertitudes de plusieurs natures. D'une part, les modifications des modalités d'acquisition et/ou de valorisation des droits à retraite se matérialisent plusieurs années plus tard : les effets restent incertains jusqu'au départ à la retraite des assurés, alors qu'ils persistent jusqu'à leur décès voire celui de leur conjoint via la réversion. L'incertitude est d'autant plus grande que la complexité des règles ne permet pas toujours d'apprécier le sens des effets produits et la population concernée. L'ampleur des effets de la réforme de 1993 (BRIDENNE, BROSSARD, 2008) a ainsi certainement longtemps été occultée par la complexité des mécanismes en jeu, touchant au calcul du salaire de référence, et à l'étalement de la réforme sur quinze ans. De ce point de vue, l'incertitude est moindre dans les régimes complémentaires que dans le régime général, car la valeur du point de retraite est un paramètre du régime qui ne dépend ni de la carrière des assurés ni véritablement de leur comportement de départ à la retraite ${ }^{27}$. Une autre source d'incertitude porte sur les droits futurs et leur valorisation à la retraite, lesquels dépendent des comportements d'activité et de départ à la retraite, ainsi que

27. L'âge de liquidation des droits dans les régimes complémentaires n'affecte pas la valeur de service du point si l'assuré justifie du taux plein au régime général, sans quoi s’appliquent des coefficients de minoration. 
des conditions macroéconomiques relatives à l'évolution des salaires, des prix, de la population active, qui déterminent de façon plus ou moins directe les barèmes législatifs et réglementaires d'acquisition et de valorisation des droits (salaire validant un trimestre, salaire plafond, valeurs d'achat et de service du point, etc.). Enfin, à cette incertitude temporelle s'ajoute parfois une information incomplète sur les droits à retraite des assurés. Si ceux-ci peuvent être relativement bien suivis au fil de leur acquisition par le régime qui les accorde, les droits accordés par les autres régimes, qui entrent dans le calcul de la retraite à travers la durée d'assurance, sont parfois plus difficiles à consolider. Les progrès récents dans la mise en place de données interrégimes devraient cependant lever en grande partie cet obstacle.

Le décalage entre l'entrée en vigueur des mesures et la matérialisation de leurs effets a probablement retardé l'adaptation des outils d'évaluation, comme les modèles de projections financières. En raison des limitations des capacités de calcul, les efforts se sont souvent concentrés sur des raisonnements en moyenne, fondés sur des données en coupe, plutôt que sur des analyses longitudinales et en dispersion qui auraient permis de saisir les transformations des carrières en train de s'opérer. L'idée d'une homogénéité des situations individuelles a ainsi longtemps régi les outils d'aide à la décision, les situations s'écartant de la norme étant jugées mineures ou mal appréhendées. En rendant impossible « toute représentation continue et prévisible du déroulement de la vie »(GuillemARD, 1993, p. 279), le brouillage des repères d'âge collectifs d'entrée et de sortie du marché du travail a soulevé une difficulté majeure pour les régimes de retraite contributifs, celle d'anticiper les recettes et charges futures et de réaliser les arbitrages entre générosité et viabilité financière. Ainsi, à défaut de pouvoir évaluer tous les effets des mesures, leur calibrage a parfois pu reposer uniquement sur des critères normatifs, comme celui de limiter les pénalités en termes d'acquisition de droits des assurés aux carrières éloignées de la carrière standard. En imposant d'attendre le départ à la retraite de cohortes complètes de bénéficiaires pour avoir une juste appréciation de la réalité de leur situation et confronter les prévisions aux réalisations, la difficulté à mesurer la progression des droits accumulés a sûrement retardé la prise de conscience par les acteurs de la politique de retraite de certaines insuffisances du système, différant d'autant la mise en place de mesures correctrices. Les règles de calcul appliquées aux assurés relevant de plusieurs régimes de retraite de base, polypensionnés, en sont un exemple.

\section{Une évolution désormais guidée par une réflexion permanente et ouverte}

Au cours des années 2000-2010, l'évaluation, l'information et le débat publics deviennent des éléments constitutifs de l'évolution du système de retraite ${ }^{28}$. Il s'agit de parvenir à un constat partagé sur la situation, de clarifier les enjeux, de faire converger des orientations et de faciliter ainsi l'acceptation des changements, voire l'adhésion

28. L'enrichissement des lois de financement de la Sécurité sociale par des indicateurs d'objectifs et de résultats, et des études d'impact témoigne aussi de cette évolution. 
sociale $^{29}$. Le COR, mis en place en 2000, est un instrument central de cette nouvelle gouvernance, lieu d'échanges et de dialogue entre divers acteurs de la politique de retraite : experts, parlementaires, représentants de l'État, des organisations professionnelles et syndicales, des associations familiales, des retraités et des personnes âgées. De façon continue, y est conduite une réflexion de fond sur les missions du système en parallèle d'une expertise en matière de retraite. Les travaux du COR permettent ainsi de stimuler le développement d'outils statistiques, modèles et indicateurs, et d'approfondir la compréhension des mécanismes du système et la connaissance de la population assurée. Au-delà des orientations politiques suivies par les pouvoirs publics successifs, les diverses réformes et révisions intervenues depuis 2003 ont pu s'appuyer sur ces analyses. En particulier, plusieurs ajustements ont été réalisés pour corriger certaines des défaillances du système jusqu'alors mal identifiées ou laissées de côté. Ainsi, les révisions des modalités d'octroi et de calcul du minimum contributif en 2004, 2009 et $2011^{30}$ ou encore des règles de calcul du salaire de référence en 2013 font directement écho aux travaux du COR qui ont pointé le manque de ciblage du minimum contributif au regard de son objectif d'assurer une pension minimum au terme d'une carrière complète (COR, 2007) et les iniquités de traitement envers les polypensionnés (COR, 2011).

Le système de retraite français actuel couvre une grande diversité de risques de carrière comparativement à d'autres pays (GERACI, 2011). Des situations de non-validation persistent toutefois et concernent davantage les assurés aux salaires modestes $^{31}$. Toutes n'ont pas vocation à être prises en charge, par exemple lorsqu'elles résultent d'un choix libre et éclairé de l'assuré d'être en retrait du marché du travail, mais d'autres interrogent sur le périmètre des risques à couvrir par un système fondé sur l'appartenance socioprofessionnelle. Les périodes d'études, par exemple, ont donné lieu à de nouvelles prises en charge. Enfin, des droits existants, dont la pertinence et l'efficacité sont discutables, ont fait l'objet de pistes de réforme. C'est le cas de certains droits familiaux, même si la réflexion menée au COR en 2008 (CoR, 2008) est encore largement ouverte (FRAGONARD et al., 2015). Ces révisions des règles, qui visent à améliorer la couverture des risques de pertes de droits à retraite, ne se font pas sans questionner la cohérence avec les autres missions du système. En particulier, modifier les règles de détermination des droits à retraite pose, par essence, des questions d'équité intergénérationnelle. Dans la mesure où les générations successives connaissent des contextes différents tout au long de leur vie, l'objectif de leur accorder un juste traitement ne peut viser une égalité de leurs situations sans entrer en contradiction avec une

29. Sur la fonction d'apprentissage dans l'élaboration des politiques publiques, voir HaLl (1993). Sur la réforme des retraites de 2003, voir TOMPSON (2010).

30. Pour favoriser les assurés obtenant le taux plein au terme d'une carrière longue et non du seul fait de leur âge, depuis 2004, une majoration est attribuée au prorata des trimestres cotisés et depuis 2010, cette majoration est limitée aux personnes ayant au moins 120 trimestres cotisés. Depuis 2012, pour que le minimum contributif bénéficie aux assurés ayant de petites retraites tous régimes confondus et non au seul régime général, il est attribué dans la limite d'un plafond de pension totale fixé à environ $90 \%$ du Smic net.

31. Voir par exemple le document 11 de la séance du COR du 25 mai 2011 déjà citée. 
égalité de traitement ${ }^{32}$. Un compromis entre équités intra- et intergénérationnelle peut alors consister à adapter les règles du système en préservant les droits des générations les plus avancées en âge tout en privilégiant des réformes paramétriques et progressives sur un rythme générationnel.

La multiplicité des objectifs attachés au système nécessite de les évaluer - et de les penser - de façon « conjointe et articulée » (CoR, 2014, p. 5). En 2014, la mise en place par le législateur d'un suivi continu du système reposant sur l'examen annuel d'une batterie d'indicateurs est révélatrice de la prise en compte de cette injonction dans la conduite de la politique de retraite. Certains de ces indicateurs sont définis par décret, d'autres sont présentés à l'initiative du COR. S'ils paraissent indispensables pour clarifier les enjeux auprès des différents acteurs de la politique de retraite, le risque serait qu'ils réduisent et norment les termes du débat en édifiant une "politique des indicateurs » (SALAIS, 2004). La définition d'indicateurs ne doit pas figer la réflexion sur leur pertinence au regard des objectifs que l'on a assignés au système, ni réduire la portée des objectifs aux indicateurs censés les évaluer. Comme le dit justement Annie FouQuet (2010, p. 317), « l'indicateur éclaire une portion de la réalité comme le réverbère son cône de lumière : le risque est de ne chercher ses clés que sous le réverbère ». La démarche analytique menée au sein du COR et la publicité de ses travaux, évaluations et réflexions, peuvent éviter cette dérive instrumentale. L'enjeu est de ne pas négliger certains objectifs au profit d'autres et de laisser s'imposer des perceptions partielles de la situation pour, au contraire, garder une vision ouverte et renouvelée des évolutions que doit prendre en charge le système de retraite. La mise en place d'un jury citoyen pour accompagner les travaux du Comité de suivi des retraites, créé par la réforme de 2014 pour rendre un avis sur l'adéquation du système à ses objectifs, témoigne déjà d'une conscience de l'apport que peut représenter une démocratisation de la gouvernance (LASCOUMES, 1996 ; DAHL, 2000).

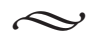

L'évolution sur longue période des règles d'acquisition et de valorisation des droits à retraite des salariés du secteur privé témoigne d'une adaptation aux grandes évolutions socio-économiques, mais aussi d'incohérences au regard des objectifs visés. Le parcours professionnel a longtemps été pensé comme une trajectoire continue, sans aléas, de la sortie du système scolaire au départ en retraite. Cette image constituait à la fois une référence pour la définition des paramètres du système de retraite et un outil conceptuel simple. Pourtant, dès les années 1970, le décalage entre la norme et la réalité est manifeste. Dans la poursuite de l'objectif d'une amélioration du niveau général des pensions, plusieurs dispositifs de compensation des aléas de carrière sont développés.

32. Par exemple, compte tenu de l'allongement de l'espérance de vie au fil des générations, une égalité d'espérance de retraite entre les générations récentes et leurs aînées suppose un relèvement de l'âge effectif de départ à la retraite et donc des paramètres d'âge et/ou de durée. L'égalité de situation entre les générations récentes et leurs aînées suppose donc de fait de les traiter différemment. 
À partir des années 1990, l'objectif de parité de niveau de vie entre actifs et retraités est en passe d'être atteint et devant l'impossibilité de pouvoir assurer financièrement un traitement équivalent aux générations futures, l'attention se tourne vers un partage plus équilibré entre actifs et retraités, visant à davantage préserver les actifs et leurs droits futurs. Un changement de paradigme s'opère alors, privilégiant une vision à long terme : l'équité intergénérationnelle se conçoit dès lors, non pas seulement entre classes d'âges, mais aussi - et surtout - entre générations dans une perspective de cycle de vie. Le tournant des années 2000 marque véritablement la mise en œuvre d'une nouvelle façon de penser l'évolution du système de retraite, plus intégrée au contexte socio-économique et démographique et à ses transformations.

En réponse à la déstandardisation et déstabilisation des carrières, les ajustements successifs du système ont conduit à desserrer le cadre normatif autour des départs à la retraite, à renforcer le lien entre carrière et retraite au cœur de la formule de calcul des pensions et à développer en contrepartie des dispositifs pour atténuer les effets des discontinuités de carrière. Le relâchement de certains paramètres comme les bornes d'âge a été réalisé de façon maîtrisée, en multipliant les critères d'éligibilité afin de conserver les comportements individuels sur une trajectoire financièrement soutenable. Cette évolution apparaît comme le fruit d'un apprentissage, tenant à la fois à une meilleure connaissance des résultats produits par le système et à une clarification des objectifs. Elle a certainement été permise par la réduction de l'incertitude temporelle, inhérente aux politiques de retraite, sous l'effet de l'arrivée à maturité du système et d'un développement important de l'expertise. Les inefficacités qui subsistent au regard de l'objectif d'assurer un traitement équitable des assurés peuvent trouver une explication dans la situation d'entre-deux actuelle où les logiques de contributivité et de compensation n'ont pas été poussées à leur terme. Ainsi, la formule de calcul conserve de nombreuses non-linéarités qui peuvent générer des effets antiredistributifs, et les dispositifs explicites de solidarité ne corrigent pas complètement les discontinuités de carrière salariale.

Le système uniformisant originel a laissé progressivement place à un système plus flexible, s'adaptant plus spontanément aux changements s'opérant sur le marché du travail. En 2017, le calcul de retraite unique pour les assurés relevant du régime général et des régimes alignés sur lui (salariés agricoles, artisans et commerçants) permet de franchir une nouvelle étape dans la réduction des iniquités à l'égard de certains assurés polypensionnés, tout en simplifiant un système encore obscur. L'essor de l'économie numérique et, avec lui, le développement de la multiactivité et du salariat par intermittence rendent encore plus indispensables des évolutions en ce sens. 


\section{BIBLIOGRAPHIE}

Albert C., Oliveau J.-B. (2011), « Simulation d'un passage du régime général en comptes notionnels à l'aide du modèle de projection Prisme », Retraite et société, nº 60, pp. 137-171.

Amossé T. (2002), « Vingt-cinq ans de transformation des mobilités sur le marché du travail », in Institut national de la statistique et des études économiques (Insee), Données sociales, Paris, Insee, pp. 235-242.

Aubert P. (2012), «Impacts directs et indirects des systèmes de retraite sur l'emploi des seniors : résultats récents », Revue française des affaires sociales, $\mathrm{n}^{\circ}$ 4, pp. 14-39.

Aubert P. (2015), « La modulation du montant de pension selon la durée de carrière et l'âge de la retraite : quelles disparités entre assurés ? », Document de travail, $n^{\circ}$ G2015/10, Paris, Insee.

Aubert P., Bachelet M. (2012), « Disparités de montant de pension et redistribution dans le système de retraite français », in Insee, L'Économie française. Comptes et dossiers. Rapport sur les comptes de la Nation 2011, Paris, Insee, coll. « Insee Références », pp. 45-62.

AuBERT P., CRÉPON B. (2003), « La productivité des salariés âgés : une tentative d'estimation », Économie et statistique, $\mathrm{n}^{\circ} 368$, pp. 95-119.

Aubert P., Duc C. (2011), « Les conséquences des profils individuels des revenus d'activité au long de la carrière sur le niveau des pensions de retraite », Économie et statistique, ${ }^{\circ}$ 441-442, pp. 159-186.

BAC C. (2011), «Articulation entre les branches famille et retraite : une illustration avec l'AVPF », Retraite et société, n ${ }^{\circ} 61$, pp. 167-183.

Behaghel L., CARoli È., Roger M. (2010), « Départ des travailleurs âgés, formation continue et changements techniques et organisationnels », Travail et Emploi, n 121, pp. 7-20.

BisSERET N. (1979), «Un coup de force : la retraite des femmes à 60 ans », Questions féministes, $\mathrm{n}^{\circ} 5$, pp. 73-106.

Blanchet D., Mahieu R. (2004), "Estimation Models of Retirement Behavior on French Data", in Gruber J., Wise D. A. (eds), Social Security Programs and Retirement around the World. Micro-Estimation, Chicago, University of Chicago Press, pp. 235-284.

BRIARD K., LELIEUR V. (2007), « Taux de rendement de l'opération retraite et redistribution intragénérationnelle », Retraite et société, $\mathrm{n}^{\circ} 51$, pp. 242-273.

BRIARD K., MAHFOUZ S. (2011), « Modulations de la retraite selon l'âge de départ : principes directeurs et évolutions depuis les années 1980 », Économie et statistique, n 441-442, pp. 15-38.

BRIDENNE I., BRosSARD C. (2008), « Les effets de la réforme de 1993 sur les pensions versées par le régime général », Retraite et société, $\mathrm{n}^{\circ}$ 54, pp. 121-153.

Brocas A.-M. (2004), « Les femmes et les retraites en France : un aperçu historique », Retraite et société, $\mathrm{n}^{\circ} 43$, pp. 11-33.

Brocas A.-M., Duriez M., Durin F., WAHL S. (1992), L'Évolution des systèmes de protection sociale : état de la recherche en France et en RFA, Paris, Centre d'information et de recherche sur l'Allemagne contemporaine (Cirac). 
Caussat L. (1996), «Retraite et correction des aléas de carrière », Économie et statistique, n $291-292$, pp. 185-201.

COE (CONSEIL D'ORIENTATION POUR L'EMPLOI) (2009), Rapport sur les trajectoires et les mobilités professionnelles, septembre.

Colin C., Mette C. (2003), « Impact des différents aléas de carrière sur les retraites : inactivité, chômage, travail à temps partiel et préretraite », Retraite et société, n⿳0 40, pp. 21-51.

COR (CONSEIL D'ORIENTATION DES RETRAITES) (2007), Retraites : 20 fiches d'actualisation pour le rendez-vous de 2008, Cinquième rapport, novembre, Paris, La Documentation française.

CoR (2008), Retraites : droits familiaux et conjugaux, Sixième rapport, décembre, Paris, La Documentation française.

CoR (2010a), Retraites : annuités, points ou comptes notionnels? Options et modalités techniques, Septième rapport, janvier, Paris, Direction de l'information légale et administrative (Dila).

COR (2010b), Retraites : perspectives actualisées à moyen et long terme en vue du rendez-vous de 2010, Huitième rapport, avril, Paris, Dila.

COR (2011), Retraites : la situation des polypensionnés, Neuvième rapport, septembre, Paris, Dila.

COR (2013), Retraites : un état des lieux du système français, Douzième rapport, janvier, Paris, Dila.

COR (2014), Évolutions et perspectives des retraites en France, Rapport annuel du COR, juin, Paris, COR.

COR - Secrétariat général (2015), « Les différences de retraite entre salariés du privé et fonctionnaires », La Lettre du COR, $\mathrm{n}^{\circ} 12$.

Courtioux P., Erhel C. (2005), «Les politiques en faveur des seniors : quelles réformes ? Comparaison Allemagne, France, Royaume-Uni, Suède », Travail et Emploi, n ${ }^{\circ} 102$, pp. 107-118.

DAhl R. A. (2000), On Democracy, New Haven, London, Yale University Press.

El Mekkaoui De Freitas N., Duc C., Briard K., Mage S., Legendre B. (2011), « Aléas de carrière et pension de retraite », Économie et statistique, $\mathrm{n}^{\circ}$ 441-442, pp. 145-158.

Feldstein M., Siebert H. (eds) (2002), Social Security Pension Reform in Europe, Chicago/ London, University of Chicago Press.

FouQuet A. (2010), «L'usage des statistiques : de l'aide à la décision à l'évaluation des politiques publiques », Revue française des affaires sociales, $n^{0} 1-2, \mathrm{pp} .307-322$.

FourCADE B. (1992), «L'évolution des situations d'emploi particulières de 1945 à 1990 », Travail et Emploi, $\mathrm{n}^{\circ}$ 52, pp. 4-19.

FouRniER J.-Y., Givord P. (2001), « La réduction des taux d'activité aux âges extrêmes, une spécificité française ? », Document de travail, $\mathrm{n}^{\circ}$ G2001/16, Paris, Insee. 
Fragonard B., Gonzalez L. (collab.), Marc C. (collab.), Fillion S. (collab.) (2015), Les Droits familiaux de retraite, Rapport, Paris, Haut Conseil de la famille.

GERACI M. (2011), «Le lien entre pension et revenus d'activité : une comparaison entre les principaux pays de l'OCDE », Économie et statistique, n $^{\circ} 441-442$, pp. 187-203.

Guillemard A.-M. (1993), « Emploi, protection sociale et cycle de vie : résultats d'une comparaison internationale des dispositifs de sortie anticipée d'activité », Sociologie du travail, vol. $35, \mathrm{n}^{\mathrm{o}} 3$, pp. 257-284.

Hall P. (1993), "Policy Paradigms, Social Learning and the State Case: The Case of Economic Policymaking in Britain", Comparative Politics, vol. 25, n 3, pp. 275-296.

Hourriez J.-M., Roux V. (2001), « Vue d'ensemble des inégalités économiques », Document de travail, $\mathrm{n}^{\circ} \mathrm{F} 0103$, Paris, Insee.

Jolivet A. (2002), « La politique européenne en faveur du vieillissement actif », Retraite et société, $\mathrm{n}^{\mathrm{o}} 36$, pp. 137-157.

LAGARde F., Worms G. (1978), « La redistribution : une problématique nouvelle », Statistiques et études financières, $\mathrm{n}^{\mathrm{O}} 32$, pp. 54-74.

LASCOUMES P. (1996), «Rendre gouvernable : de la "traduction" au "transcodage". L'analyse des processus de changement dans les réseaux d'action publique », in Centre universitaire de recherches administratives et politiques de Picardie (Curapp), La Gouvernabilité, Paris, Presses universitaires de France, pp. 325-338.

Muller P. (2000), « L'analyse cognitive des politiques publiques : vers une sociologie politique de l'action publique », Revue française de science politique, vol. 50, n ${ }^{\circ}$, pp. 189-207.

PIERSON P. (2000), "Increasing Returns, Path Dependence, and the Study of Politics", American Political Science Review, vol. 94, n 2, pp. 251-267.

Salais R. (2004), « La politique des indicateurs. Du taux de chômage au taux d'emploi dans la stratégie européenne pour l'emploi », in Zimmermann B. (dir.), Les Sciences sociales à l'épreuve de l'action. Le savant, le politique et l'Europe, Paris, Éditions de la Maison des sciences de l'homme, pp. 287-331.

Streeck W., Thelen K. (2005), "Introduction”, in Streeck W., Thelen K. (eds), Beyond Continuity: Institutional Change in Advanced Political Economies, Oxford, Oxford University Press, pp. 3-39.

Tompson W. (2010), « France : la réforme des retraites de 2003 », in Tompson T., Price R. (collab.), L'Économie politique de la réforme : retraites, emplois et déréglementation dans dix pays de l'OCDE, Paris, Éditions OCDE, pp. 87-110.

Troadec T. (1998), « L’âge des extrêmes », Retraite et société, n 24, pp. 7-27.

WALRAET E., VinCENT A. (2003), « La redistribution intragénérationnelle dans le système de retraite des salariés du privé : une approche par microsimulation », Économie et statistique, $\mathrm{n}^{\mathrm{o}} 366$, pp. 31-56.

WitT U. (2003), "Economic Policy Making in Evolutionary Perspective", Journal of Evolutionary Economics, vol. 13, n ${ }^{\circ}$ 2, pp. 77-94. 